(D)

CORPUS PUBLISHERS

\section{Advance Research in}

\section{Organic and Inorganic}

Chemistry (AROIC)

\section{Volume 3 Issue 1, 2022}

Article Information

Received date : January 26, 2022

Published date: February 14, 2022

*Corresponding author

T. Mavromoustakos, National and

Kapodistrian University of Athens,

Department of Chemistry, Laboratory of

Organic Chemistry, Panepistimioupolis

Zografou, Athens

\section{Keywords}

Cinnamic Analog; Synthesis;

Spectroscopy; Molecular Dynamics

DOI:10.54026/AROIC/1006

Distributed under Creative Commons CC-BY 4.0

\title{
Spectroscopic and Computational Study of the Ugi Cinnamic Adduct NGI25
}

N Georgiou' ${ }^{1}$, N Gouleni' ${ }^{1}$ E Chontzopoulou ${ }^{1}$, G S Skoufas ${ }^{1}$, A Gkionis ${ }^{1}$, D Tzeli ${ }^{2,3}$, S Vassiliou ${ }^{1}$, T Mavromoustakos $^{{ }^{*}}$

${ }^{1}$ National and Kapodistrian University of Athens, Department of Chemistry, Laboratory of Organic Chemistry, Panepistimioupolis Zografou, 11571, Athens

${ }^{2}$ National and Kapodistrian University of Athens, Department of Chemistry, Laboratory of Physical Chemistry, Panepistimioupolis Zografou, 11571, Athens

${ }^{3}$ Theoretical and Physical Chemistry Institute, National Hellenic Research Foundation, 48 Vassileos Constantinou Ave, Athens 116 35, Greece

\section{Abstract}

The structure assignment and conformational analysis of cinnamic derivative N-benzyl-N-(2-(cyclohexylamino)-2-oxoethyl) cinnamamide (NGI25) was carried out through Nuclear Magnetic Resonance (NMR) spectroscopy, Molecular Dynamics (MD) and Quantum Mechanics (QM), i.e., semiempirical and Density Functional Theory (DFT) calculations. Specifically, homonuclear (2D-COSY, 2D-NOESY) and heteronuclear (2D-HSQC, 2D-HMBC) spectra were obtained. Through them, the resonant values of the hydrogens and their constituent carbons were identified. After structure identification, NGI25 was subjected to computational calculations to reveal its most favorable conformations. QM, MD were in agreement with the spatial correlations that were observed in 2D-NOESY spectra.

Introduction

In this article Ugi-4R reaction was used to synthesize cinnamic adduct NGI25 [1,2].

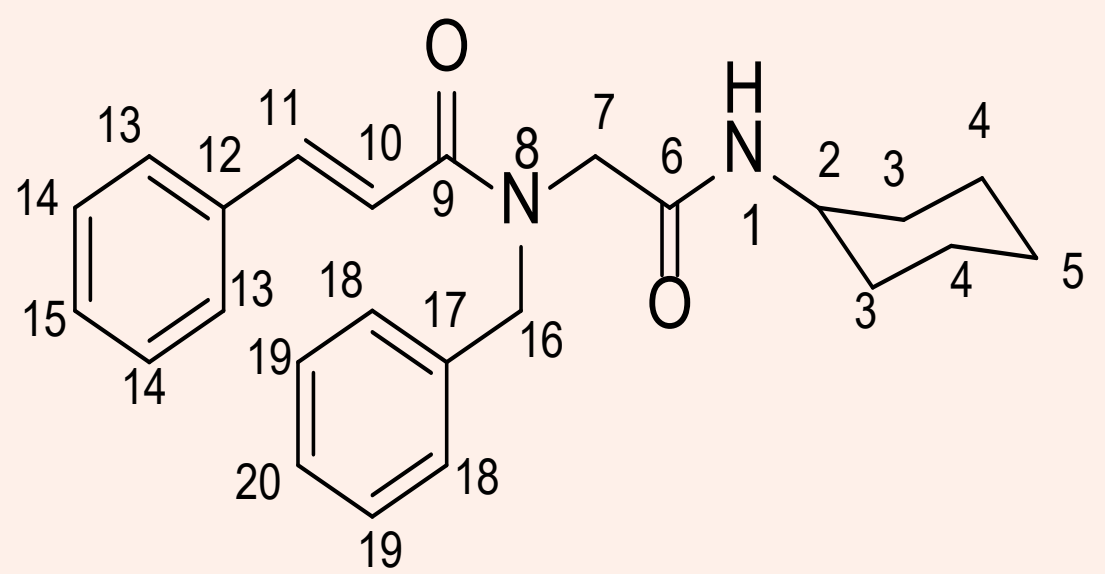

Figure 1: $N$-benzyl- $N$-(2-(cyclohexylamino)-2-oxoethyl) cinnamamide, NGI25

In (Figure 1) Structure of NGI25 with carbons numbered as these are used in the assignment of NMR spectra Structure elucidation [3] was performed through NMR experiments and specifically, 1D and 2D experiments. A detailed conformational analysis was carried out via DFT [3,4] and MD [5]. These methods are the most appropriate for these organic compounds. A wide range of conformations has been examined through PM6 [6] and DFT calculations [7]. Finally, molecular dynamics calculations were used to study the mobility of NGI25 in DMSO.

Results

As a convenient starting point, the structure assignment was started by the protons $\mathrm{H}-10$ and $\mathrm{H}-11$. The final part was to identify the quaternary and carbonyls carbons through 2D-HMBC experiment. On the next stage, conformational analysis was carried out to find the most favorable configuration for NGI25. NGI's conformations do not present important differences between the results of all the computational methods and experimental NMR results. In particular, the conformations provided by Grid Scan, MD, PM6 and DFT methodology are quite similar (see Figure 3), and only minor differences between the 
conformers that derived from PM6 and DFT calculations are observed.

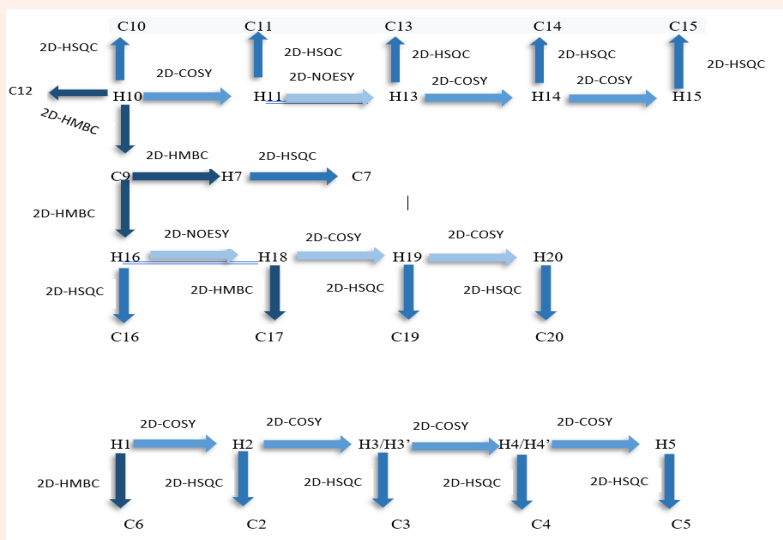

Figure 2: Overall diagram showing the identification strategy of the NGI25 compound in DMSO solvent.
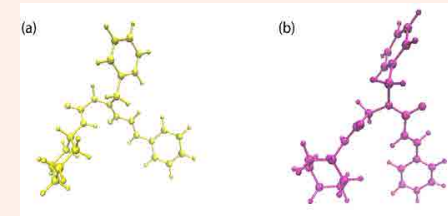

(c)
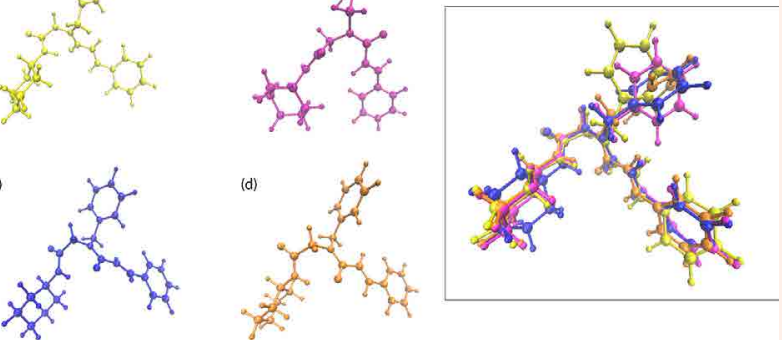

- Grid Scan

- PNG

Figure 3: Conformation of NGI25 derived from (a) Grid scan (b) Molecular Dynamics (c) PM6 (d) DFT (B3LYP/6-311+G(d,p)) methodologies. The superimposition of these conformers is illustrated in the framed part of the figure.

\section{Conclusion}

Briefly, our findings demonstrate that cinnamic adducts are very important compounds and in the future they will be used for molecular docking experiments to find some possible biological targets.

Acknowledgments: Materials were supported by Special Account for Research Grants (SARG), National Kapodistrian University of Athens (NKUA).

Conflicts of Interest: The authors declare no conflict of interest.

\section{References}

1. Fouad MA, Hamid AH, Ayoup MS (2020) Two decades of recent advances of ugi reactions: Synthetic and pharmaceutical applications. RSC Adv 10(70): 4264442681

2. Ruijter E, Orru RVA (2013) Multicomponent Reactions-Opportunities for the
Pharmaceutical Industry. Drug Discov Today Technol 10 (1): e15-e20.

3. Imtiaz S, Muzaffar S, Ali SM (2021) Demonstrating Accuracy of the Already Proposed Protocol for Structure Elucidation of Cyclodextrin Inclusion Complexes by Validation Using Quantitative ROESY Analysis. J Incl Phenom Macrocycl Chem 100 (1-2): 71-87.

4. Ando S (2018) Efficient Hybrid Functional and Basis Set Functions for DFT Calculation of Refractive Indices and Abbe Numbers of Organic Compounds. Chem Lett 47 (12): 1494-1497.

5. Martyna GJ, Tobias DJ, Klein ML (1994) Constant Pressure Molecular Dynamics Algorithms. J Chem Phys 101: 4177-4189.

6. Becke AD (1993) A New Mixing of Hartree-Fock and Local Density-functiona Theories. J Chem Phys 98(2): 1372-1377.

7. Bütikofer A, Løken KV, Salvanes KG (2019) Infant Health Care and Long-Term Outcomes. The Review of Economics and Statistics 101(2): 341-354. 